\title{
Research on regional differences of the impact of clean energy development on carbon dioxide emission and economic growth
}

Weiping Jia ${ }^{1 凶}$, Xianwen Jia ${ }^{1}$, Ling Wu${ }^{1}$, Yanbing Guo ${ }^{1}$, Teng Yang ${ }^{1}$, Ermei Wang ${ }^{1} \&$ Pan Xiao ${ }^{1}$

\begin{abstract}
"China is by far the world's largest importer of oil and emitter of carbon dioxide." Therefore, clean energy development (CED) is of great practical significance to reduce carbon dioxide emission (CDE), ensure energy security, and achieve green economic growth. What is the role of CED in reducing CDE? Can CED, which requires significant investment, promote economic growth? For the above aims, according to the panel data of 30 provinces composed of accurate data during 1979 to 2016 and prediction data from 2017 to 2030 in China, this research employs "a non-parametric and additive regression model" to explore the linear and nonlinear influence of CED on CDE and economic growth. The results show that CED does not play an essential role in reducing $C D E$ and fostering economic growth from a linear perspective; the influence of CED on CDE and economic growth in China's western, central and eastern regions is significantly different from a nonlinear perspective. Hence, the Chinese government ought to fully play the critical role of clean energy in reducing $C D E$ and fostering economic growth.
\end{abstract}

\footnotetext{
${ }^{1}$ Hunan University of Arts and Science, Changde, Hunan, China. ${ }_{\text {email: 646509255@qq.com }}$
} 


\section{Introduction}

ince the economic system reform, China's GDP has grown by an average of $15 \%$ annually, which is driven by industrialization and urbanization from 1979 to 2016 (Lin et al., 2017). Since 2010, China has become "the world's second-largest economy after the United States" ("National Bureau of Statistics of China," 2019). Yet China's long-term economic growth is characterized by the extensive development of low output, high input, and high consumption (Chen, 2009; Yang and Poon, 2009). In the process of rapid economic growth, "a lot of fossil fuels is consumed, and a lot of carbon dioxide is emitted" (World Bank, 2019). "The largest emitter of carbon dioxide and the largest energy consumer is China in the world in 2006 and 2011, respectively" (Yang et al., 2016; Lin and Zhu, 2017; Nan et al., 2019; Shao et al., 2019a; Shao et al., 2019b; Yang and Yang, 2019).

The solemn "environmental issues" are aroused by a lot of $\mathrm{CDE}$, which has made the Chinese government realize in recent years that it must formulate a series of environmental policies to promote the CED (Costanza et al., 2014; Wen and Shao, 2019; Nan et al., 2019; Wu and Han, 2020). Therefore, "the central and local governments at all levels actively expand the investment in CED" (Zeng et al., 2018; Li et al., 2019; Tian et al., 2019), "rapidly increasing from 8.15 billion RMB yuan in 2008 to 59.506 billion RMB yuan in 2016, with an average annual growth rate of $28.2 \%$ " ("National Bureau of Statistics of China," 2008-2016). Therefore, the clean energy (CE) industry has been developed rapidly. "China has a vast territory, and there are significant differences in the industrial structure, resource endowment, technological level, and urbanization level of various regions" (Miao et al., 2019). Hence "there are also significant differences in economic growth and CDE in China's eastern, central and western regions" (Lu and $\mathrm{Xu}, 2019$; Wang and Wang, 2019; Nie et al., 2019). "The per capita GDP of the eastern region was 38370.4 RMB yuan, while the per capita GDP of central and western regions were, respectively, 19,348.7 RMB yuan and 18,314.5 RMB yuan from 1997 to 2016; the industrial added value in the eastern, central, and western regions accounted for $43.7 \%, 40.4 \%$, and $41.8 \%$ of GDP from 1997 to 2016, respectively; the CDE in China's eastern, central, and western regions were, respectively, 348 million tons, 268 million tons, and 183 million tons from 1997 to 2016" ("National Bureau of Statistics of China," 1979-2016). If these differences are ignored, it is challenging to employ the outcomes drawn at the macroscopic level to different regions.

Existing research deficiencies to be remedied, this article employs "a non-parametric additive regression model" at the provincial level to study the impact of CED sources on the CDE and economic growth. Therefore, it is essential to further investigate the effect of the growth of CE sources on the CDE and economic growth from the regional disparity.

\section{Literature review}

$\mathrm{CDE}$ can meet economic growth demand, reduce $\mathrm{CDE}$, and improve the industrial structure, and realize sustainable economic growth. Therefore, a growing number of researches focus on the CED.

Research on how to develop CE sources to decrease CDE. Cao (2009) proposed that "the government could promote the rapid growth of CE production enterprises by implementing tax relief or financial subsidies, to reduce $\mathrm{CDE}$ generated by the consumption of fossil energy." Lu et al. (2013) studied "China's CDE from 1994 to 2008 and found that optimizing the energy structure can increase CE consumption and help control CDE". Hence all regions in China should reduce coal usage and strive to develop CE sources. Dogan and Seker (2016) and Zoundi (2017) used the causal test to find that "there is a two-way causal relationship between $\mathrm{CE}$ and $\mathrm{CDE}$, and the use of $\mathrm{CE}$ can reduce CDE." However, Kahia et al. (2016) employed "a panel vector autoregressive model" to explore the CE effect on CDE of 24 countries in North Africa and the middle east, and the results showed that CE usage does not significantly decrease CDE.

Research on whether CED hinders or promotes economic growth. Lin and Li (2014) thought that "the CED can accelerate the industrial structure upgrading and realize sustainable economic growth in the long term." Zhang and Liu (2015) believed that "the CED will raise energy prices in the short term, thus inhibiting investment, consumption, and economic growth; the CED will promote technological progress and capital investment in energy-saving and emission reduction in the long term, thus driving economic growth." Inglesi (2016) used "a panel data model" to investigate and found that "the CED is conducive to promoting sustainable economic growth.” Kocak and Sarkgünesi (2017) tested "the relationship between CE consumption and economic growth in the black sea and Balkan countries and found that there was a cointegration relationship between the two." The simulation results employing the "Granger test and error correction model" demonstrate that "there may be a two-way longterm and short-term causality between economic growth and CED” (Kathia et al., 2017).

The previous literature is valuable to provide references for the current study. The theoretical contribution of current research to the literature is that there is room for improvement in the study that CED promotes economic growth and decreases CDE. First, the interaction among economic variables is assumed to be linear in most of the existing literature. Still, the economic activities, in reality, are complex and changeable, and there are plenty of nonlinear interactions among many economic variables. Ignoring the nonlinear interaction among many economic variables is bound to cause estimation results with significant errors. The obtained research results cannot fully explain the complex situation in economic reality. Secondly, much current research literature mainly discusses the influence of CED on the $\mathrm{CDE}$ and economic growth from a macro-perspective.

\section{Theoretical framework}

Theoretical model. The relationship among economic variables, in reality, is not necessarily linear, and the nonlinear relationship of parameters among variables is difficult to determine. Traditional linear or nonlinear econometric models often have model parameter setting errors in practical application. Compared that to "a linear model," "the non-parametric regression model" has the advantages of free form, fewer constraints, no requirements for data distribution, strong adaptability, high robustness and so on.

"The non-parametric additive regression model" integrates the data-driven superiority of "the non-parametric regression model" and also avoids "the dimension disaster" caused by too high a dimension in "the non-parametric regression model." Stone (1985) proposed "the additive regression model." Its form is as follows:

$$
Y_{i}=\sum_{j=1}^{p} f\left(x_{i j}\right)+\mu_{i}, \mu_{i} \sim \operatorname{iid}\left(0, \sigma^{2}\right)
$$

Where $f\left(x_{i}\right)$ is "the unary non-parametric function." $f\left(x_{i}\right)$ can be reckoned using "a unary non-parametric regression model." $\mu_{i}$ is "the random error," and $E\left(\mu_{i}\right)=0, \operatorname{var}\left(\mu_{i}\right)=\sigma^{2}$. Suppose $E\left(f_{j}\right)=0(j=1,2 \ldots p)$, it is smooth. Furthermore, "the additive 
regression model" may also be represented as Eq. (2):

$$
E\left(Y_{i} \mid x_{i 1}, x_{i 2}, \ldots, x_{i p}\right)=\sum_{j=1}^{p} f\left(x_{i j}\right)
$$

To better explain the influence of CED on CDE and regional economic growth, this research adds "linear parameters" to "the additive regression model," and its form is as follows:

$$
E\left(Y_{i} \mid x_{i 1}, x_{i 2}, \ldots, x_{i p}\right)=\alpha+\sum_{j=1}^{p} \beta_{j} x_{i j}+\sum_{j=1}^{p} f_{j}\left(x_{i j}\right)
$$

Where $\alpha$ and $\beta_{j}$ are linear regression parameters, there are many evaluation means for "the additive regression model," currently the most widely used is "the back fitting algorithm" (Buja and Tibshirani, 1989).

\section{Empirical model}

CED has an impact on economic growth. Many scholars currently employ "the Solow model" to study the main factors affecting economic growth. Therefore, according to "the Solow model”, this paper constructs the model as follows:

$$
Y_{t}=A_{t} K_{t}^{\alpha} L_{t}^{\beta}
$$

Where $Y$ indicates the level of the production; $L$ indicates the input workforce; $K$ indicates the capital investment; $\alpha$ represents the contribution share of invested capital to the level of the production; $\beta$ represents the contribution share of the input labor force to the level of the production; $A$ indicates that technological progress is a constant term; time is represented by $t$. Besides, CED will help optimize the energy consumption structure of economic society, upgrade the industrial facilities, and accelerate economic green and sustainable development. Moreover, China is currently vigorously promoting urbanization, which supports "the rural surplus laborers" employment and increases output. Therefore, this paper introduces CED and urbanization into Eq. (4) to construct a model of China's economic growth. Its form is as follows:

$$
Y_{t}=A_{t} K_{t}^{\alpha} L_{t}^{\beta} \mathrm{CE}_{t}^{\beta_{1}} \mathrm{URB}_{t}^{\beta_{2}} \mu_{t}
$$

Where CE indicates the clean energy, which is obtained by multiplying the total energy production of provinces over the years by the proportion of CE total energy production in total energy production to evaluate the CE production capacity (100 million kWh). URB indicates Urbanization, which is assessed by the urban population as a percentage of the total population. Economic growth is represented by $Y$, indicated by per capita GDP. According to the characteristics of empirical analysis using panel data, this paper transforms Eq. (5) into:

$$
\begin{aligned}
\operatorname{LGDP}_{i t}= & C+\beta_{1} \mathrm{LTEC}_{i t}+\beta_{2} \mathrm{LCAP}_{i t}+\beta_{3} \mathrm{LLAB}_{i t} \\
& +\beta_{4} \mathrm{LCE}_{i t}+\beta_{5} \mathrm{LURB}_{i t}+\mu_{i t}
\end{aligned}
$$

Where $C$ represents "the constant term," $\mu$ represents "the random error term," and $i$ represents the unit number of crosssection, GDP represents economic growth, TEC represents technological progress, CAP represents capital accumulation, $\mathrm{LAB}$ represents labor input. $\mathrm{L}$ is the logarithm of the variable to eliminate possible heteroscedasticity. $\beta_{j}$ is the estimated parameter. Because the set linear parameter model is error-prone, the current study uses "a non-parametric additive regression model" to fit the influence of CED on economic growth. The form is as follows:

$$
\begin{aligned}
\mathrm{LGDP}_{i t}= & C+\beta_{1} \mathrm{LTEC}_{i t}+\beta_{2} \mathrm{LCAP}_{i t}+\beta_{3} \mathrm{LLAB}_{i t}+\beta_{4} \mathrm{LCE}_{i t} \\
& +\beta_{5} \mathrm{LURB}_{i t}+g_{1}\left(\mathrm{LTEC}_{i t}\right)+g_{2}\left(\mathrm{LCAP}_{i t}\right) \\
& +g_{3}\left(\mathrm{LLAB}_{i t}\right)+g_{4}\left(\mathrm{LCE}_{i t}\right)+g_{5}\left(\mathrm{LURB}_{i t}\right)+\mu_{i t}
\end{aligned}
$$

Where $g_{j}(j=1,2, \ldots, 5)$ is "the estimated non-parametric function".

CDE is affected by CED. Ehrlich and Holden (1971, 1972) first proposed "IPAT ( $I$ is the impact of population on the environment; $P$ is the total population; $A$ is per capita wealth; $T$ is technological progress) model" to express the effect of population pressure on the ecological environment, which is often used to discuss the affecting factors of surrounding environment change. To avoid some defects of the "IPAT model," York et al. (2003) put forward the "Stochastic Impacts by Regression on Population, Affluence, and Technology (STIRPAT) model" in view of the "IPAT model." This research uses "the classical STIRPAT model" to discuss the influence elements of environmental pollution, and its primary model is as below:

$$
I_{t}=a P_{t}^{b} A_{t}^{c} T_{t}^{d} e_{t}
$$

Where $I$ is some kind of pollutant emission, the population size is represented by $P, A$ means the economic prosperity, and technical factors are represented by $T$. Besides, some literature studies show that "government environmental regulation and energy consumption structure of industry" are also key factors influencing CDE (Zhang and Liu, 2015). Consequently, to study the influence of CED on the CDE, this research enlarges Eq. (8) to construct "the affecting factors model of CDE." The logarithm of the variables in the extended model is taken to eliminate the possible heteroscedasticity. The form is as follows:

$$
\begin{aligned}
\mathrm{LCO}_{2 i t}= & C+\beta_{1} \mathrm{LPOP}_{i t}+\beta_{2} \mathrm{LGDP}_{i t}+\beta_{3} \mathrm{LEI}_{i t}+\beta_{4} \mathrm{LCE}_{i t} \\
& +\beta_{5} \mathrm{ENS}_{i t}+\beta_{6} \mathrm{LER}_{i t}+\mu_{i t}
\end{aligned}
$$

Where $C$ indicates "the constant term," $\mathrm{CO}_{2}$ indicates $\mathrm{CDE}$, and the total population is characterized by POP. GDP indicates the economic prosperity, which is evaluated by per capita GDP; EI indicates the energy intensity, which is used to measure the technical progress level; the clean energy is characterized by CE; ENS is "the energy consumption structure"; ER indicates the environmental regulation; $\beta_{j}$ indicates the parameter to be estimated. According to the variables determined by Eq. (9) and the theory of Eq. (3), "the non-parametric additive regression model of CDE" is constructed, the form is as follows:

$$
\begin{aligned}
\mathrm{LCO}_{2 i t}= & C+\beta_{1} \mathrm{LPOP}_{i t}+\beta_{2} \mathrm{LGDP}_{i t}+\beta_{3} \mathrm{LEI}_{i t}+\beta_{4} \mathrm{LCE}_{i t} \\
& +\beta_{5} \mathrm{ENS}_{i t}+\beta_{6} \mathrm{LER}_{i t}+g_{1}\left(\mathrm{LPOP}_{i t}\right)+g_{2}\left(\mathrm{LGDP}_{i t}\right) \\
& +g_{3}\left(\mathrm{LEI}_{i t}\right)+g_{4}\left(\mathrm{LCE}_{i t}\right)+g_{5}\left(\mathrm{ENS}_{i t}\right)+g_{6}\left(\mathrm{LER}_{i t}\right)+\mu_{i t}
\end{aligned}
$$

Where $g(\cdot)$ is "the non-parametric function," the rest of Eq. (10) is the same as Eq. (9).

Variables selection and data sources. This research employs "a non-parametric additive regression model" in studying the influence of CED on CDE and regional economic growth.

Variable selection for economic growth. The dependent variable of CED to the economic growth model is economic growth. Many current studies use an average per person GDP to evaluate economic growth. This research also applies the average per person GDP in provinces to indicate economic growth (yuan) in ones. 
To clarify the effect of price factors, this research involves the average per person GDP deflation index to deflate average per person GDP. There are five independent variables: the first is CE. The total amount of energy produced by provinces and CE (e.g., hydropower, nuclear energy, wind energy, geothermal energy, etc.) as a percentage of total energy production comes from "the provincial statistical yearbook and China energy statistical yearbook." The second is urbanization, and it is evaluated by "urban population as a percentage of the total population." The third is labor input, represented by "the total number of employees." Labor input refers to the amount of labor invested in production. According to Qiu et al. (2020), the amount of work can be measured by the number of employees. The fourth is capital investment, which uses "the perpetual inventory method" to measure "the capital stock of sample period" (Pokki et al., 2018). The fifth is technological progress. This research applies "the Malmquist productivity index method" to calculate "total factor productivity" to measure "technological progress".

Variable selection of CDE. The dependent variable of CED to the CDE model is CDE. The "total energy consumption (tons of standard coal)" of provinces, and coal, petroleum, CE, and natural gas as a ratio of "total energy consumption," comes from "the statistical yearbook" of provinces. "The UN Intergovernmental Panel on Climate Change (IPCC)," "the International Energy Agency (IEA)," and the US "energy information administration (EIA)" announced that "the carbon dioxide emission factor" of "a ton of standard coal" is between 2.68 and 2.72 tons. In this paper, "the carbon dioxide emission factor" of "a ton of standard coal" is set at 2.70 tons. This research uses "the total consumption of fossil energy" (e.g., coal, natural gas, and petroleum) transformed into "tons of standard coal" multiplied by "the carbon dioxide emission factor" of "tons of standard coal" to achieve the total CDE. There are five independent variables. The first is CE, the same as CE in the economic growth model. The second is the total population, which indicates the total population size. The third is economic growth, which is evaluated in average per person GDP. This research eliminates the impact of price elements using average per person GDP deflator price. The fourth is energy intensity (EI), which evaluates the technological level through energy consumption divided by GDP. The fifth is "the energy consumption structure," which is indicated in coal consumption as a ratio of "total energy consumption." The sixth is the environmental regulation index, constructed by two indicators: sulfur dioxide emission per unit and dust and smoke emission per unit.

Data sources. The sample period of this paper is composed of two parts from 1979 to 2030 . The first part is from 1979 to 2016, data source of average per person GDP, average per person capita GDP deflation index, urban population, total population, CE production capacity, and a number of employees are all from "China Statistical Yearbook"; data source of capital stock comes from "the statistical yearbook" of provinces; data source of "energy consumption structure" comes from "China energy statistical yearbook."
The second part is from 2017 to 2030 . The data in the second sample period are predicted values. Because China's CE production is relatively small at this stage, and its percentage in "total energy production" is low. Its contribution to CDE reduction and regional economic growth may be limited. Although the share of $\mathrm{CE}$ consumption in "total energy consumption" is still small at this stage. However, according to the current economic growth, it can be predicted that the $\mathrm{CE}$ market requirement shall reach 800 million "tons of standard coal" by 2030 in China, and the percentage of "clean energy consumption" in "total energy consumption" may rise $21 \%$. The vigorous growth of $\mathrm{CE}$ consumption will significantly decrease the rate of fossil energy consumption and reduce the carbon intensity of economic growth. Moreover, "the Chinese government promised to decrease CDE per unit of GDP by $60-65 \%$ by 2030 compared to 2005, and control the growth of total CDE, and make an effort to gradually reduce carbon emission after carbon emission reaches the maximum around 2030". Therefore, to study the impact of CED on CDE reduction and economic growth, this research employs accurate data from 1979 to 2016 and the forecast data from 2017 to 2030 .

The method of data forecasting is as follows. As the data of "per capita GDP deflation index," "total factor productivity index," and "fixed asset investment price index" are comparatively stable, there is no noticeable trend of change over time, and this paper chooses "the Autoregressive Integrated Moving Average model (ARIMA)" to calculate their data. For the rest of the variables that change over time (e.g., per capita GDP, CE production, total energy consumption, industrial output value, environmental regulations, and employment number, etc.), this paper uses a double exponential model to forecast their data.

Comparing the accurate data with the forecast data from 1979 to 2016, it is found that the accurate data and the forecast data have a higher fitting degree and minor errors. This shows that the forecast method selected in this paper is suitable, so the forecast results are highly reliable from 2017 to 2030 .

\section{Empirical results}

The endogenous test. Before employing "a non-parametric additive regression model" in regression estimate, it is necessary to solve the endogenous issues arising from the possible "two-way causality" between CED and CDE and economic growth. If there is endogeneity, the estimation results will be biased and inconsistent (Shao et al., 2019b). This section will examine whether CED in the CDE model is an endogenous variable and economic growth model, respectively (see Table 1). Firstly, DWH (Wu, 1974; Hausman, 1978) is used to test the endogeneity of CE in Eq. (6). The test results of Durbin-Wu-Hausman accept the null hypothesis that CE is exogenous. Secondly, EHR weak echogenicity test method (Engle et al., 1983; Bravo and Marin, 2011) is used to test the endogeneity of CE in the CDE model (Eq. 9). The $F$-statistic value of the test is 3.79 , and the $P$-value of correspondence is 0.0516 . Under the condition of a significance level of $5 \%$, the test results accept the null hypothesis. This means that $\mathrm{CE}$ is exogenous in $\mathrm{CDE}$.

Table 1 Endogenous test: clean energy.

Original hypothesis: clean energy is exogenous; alternative hypothesis: clean energy is endogenous

The effect of clean energy on economic growth (model 6)

Wu-HausmanF text

Durbin-Wu-HausmanChi-sq text
$F(1,1492)=0.00007$

Chi-sq(1) $=0.00007$

\section{The effect of clean energy on CDE (model 9)}

Original hypothesis

$\gamma=0$
F-statistic

$F(1,1447)=3.79$
$P$-value 0.0516 
Table 2 Linear effect of CED on regional economic growth.

\begin{tabular}{|c|c|c|c|c|}
\hline Models & Variables & East region & Central region & West region \\
\hline \multirow[t]{5}{*}{ Estimation results of "linear non-parametric additive regression model" } & Intercept & $3.225^{\star \star \star}$ & $3.836^{\star \star \star}$ & $6.779^{\star \star \star}$ \\
\hline & Clean energy & $-0.109^{\star \star \star}$ & $-0.011^{\star \star}$ & $-0.077^{\star \star \star}$ \\
\hline & Urbanization & $0.846^{\star \star \star}$ & $0.719^{\star \star \star}$ & $0.147^{\star \star \star}$ \\
\hline & Capital investment & $0.104^{\star \star \star}$ & $0.080^{\star \star \star}$ & $0.256^{\star \star \star}$ \\
\hline & Technical progress & $-0.145^{\star \star}$ & 0.082 & 0.033 \\
\hline \multirow[t]{4}{*}{ Estimation results of "linear regression model" } & Intercept & $3.787^{\star \star \star}$ & $4.018^{\star \star \star}$ & $4.406^{\star \star \star}$ \\
\hline & Labor input & $0.079^{\star \star \star}$ & $0.169^{\star \star \star}$ & $0.124^{\star \star}$ \\
\hline & Capital investment & $0.114^{\star \star \star}$ & $0.169^{\star \star \star}$ & $0.236^{\star \star \star}$ \\
\hline & Technical progress & $-0.114^{\star \star}$ & -0.011 & -0.019 \\
\hline
\end{tabular}

The effect of CED on regional economic growth. In this article, "the non-parametric additive regression model" is applied to estimate the regression of "the eastern, central and western regions." Among them, Table 2 shows the results of linear estimation, and Fig. 1 shows the consequences of nonlinear analysis.

The linear impact results. The estimated results of explanatory variables for the linear part mostly passed the significance level test of $10 \%$ or higher in Table 2 . Among them, the impact factor of CED on the economic growth of "the eastern, central and western regions" are respective $-0.109,-0.011$, and -0.077 , which means that the economic growth of the three regions will decrease by $0.109,0.011$, and 0.077 percentage points for every $1 \%$ increase in CE output. The linear impact factor is negative, which means that, on average, CED hinders economic growth. This is mainly because CED needs a lot of capital investment. However, CE production enterprises often lack sufficient capital investment, which requires local governments at all levels to increase financial support and implement tax relief policies. These will increase the burden of economic growth, so the effect of CED on economic growth is negative. However, in the wake of the expansion of the CE production scale, the contribution of CED to economic growth will increase rapidly, and promote high-quality and efficient economic growth.

The results of nonlinear impact. The nonlinear impact of CED on the economic growth in "the eastern, central and western regions" is shown in Fig. 1. The nonlinear effect of CED on economic growth passed the significance level test of $1 \%$ in the eastern region. Specifically, the nonlinear impact of CED on economic growth shows a "W-shaped" curve. CED hindered economic growth in the early stage. This is mainly because a substantial capital investment in the early stage of CED is needed; for example, photovoltaic power plants, large hydropower stations, nuclear power plants, etc., are constructed. The considerable investment made it unaffordable for many companies. Therefore, these enterprises need government financial investment. In addition, to encourage the rapid development of $\mathrm{CE}$, local governments continue to implement favorable policies, for example, the reduction of income tax for CE producers. The tax reduction and exemption and investment in CE producers augment the burden of local economic development, which hinders economic growth. In the medium stage, due to the effect of some exterior elements (e.g., the price fluctuation in the international energy market affects the CE producers), the CED makes the economic growth fluctuate slightly. In the late period, as the scale of $\mathrm{CE}$ producers continues to expand, these producers not only create a lot of employment opportunities, drive the growth of
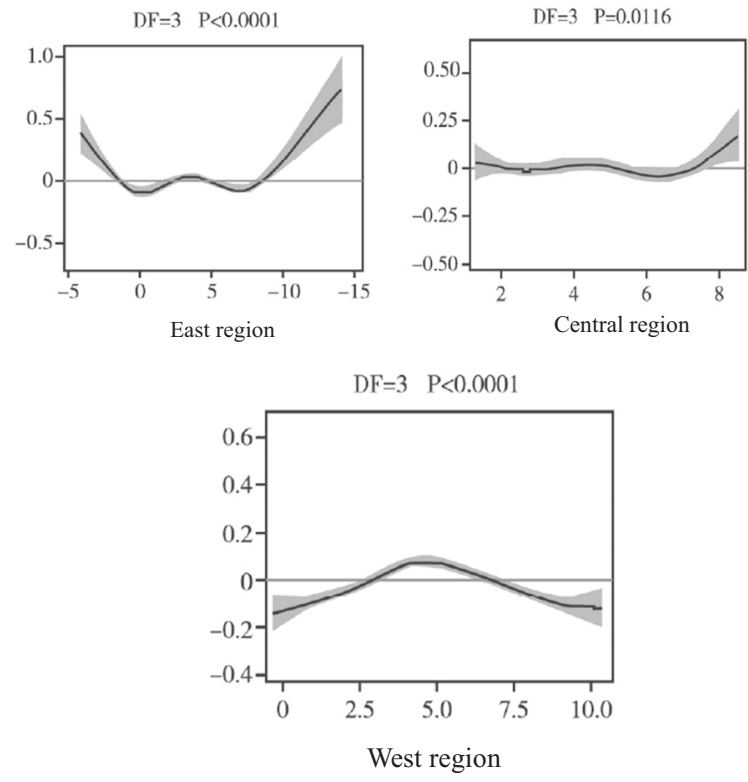

Fig. 1 The nonlinear effect of CED on China's regional economic growth. The nonlinear impact of CED on economic growth shows a "W-shaped" curve in the eastern region. The nonlinear impact of CED on economic growth shows a gentle "W-shape" curve in the central region. The nonlinear effect of CED on economic growth shows an inverse "U-shaped" curve in the western region.

relevant industries (e.g., crystalline silicon cells and modules, etc.), but also reduce production costs and product prices, and promote regional economic growth.

The nonlinear impact of CED on economic growth shows a gentle "W-shape" curve in the central region. This indicates that CED played no role in driving economic growth in earlier times. The main reason is that many energy producers need the support of government monetary funds and tax policies in the central region, which raise the cost of economic growth. CED in the medium term has little effect on economic growth. CED in the later stage promotes economic growth. Through the early and medium stage development, the production scale of CE producers in the central region is expanded. The market viability and competitiveness of these producers have been improved, and CE output is increased. Meantime, CED drives the growth of environmental protection and energy-saving industries and has become "a new economic growth point" in the central region.

The nonlinear effect of CED for the western region on economic growth appears an inverse "U-shaped" curve. This 


\section{Table 3 The linear impact of CED on regional CDE.}

\begin{tabular}{|c|c|c|c|c|}
\hline Models & Variables & East region & Central region & West region \\
\hline \multirow[t]{6}{*}{ Estimation results of linear non-parametric additive regression model } & Intercept & $-6.251^{\star \star \star}$ & $-3.810^{\star \star \star}$ & $-4.207^{\star \star \star}$ \\
\hline & Clean energy & $0.012^{\star \star \star}$ & $0.074^{\star \star \star}$ & $0.077^{\star \star \star}$ \\
\hline & Economic growth & $0.603^{\star \star \star}$ & $0.922^{\star \star \star}$ & $0.717^{\star \star \star}$ \\
\hline & Energy intensity & $0.676^{\star \star \star}$ & $0.520^{\star \star \star}$ & $0.3623^{\star \star \star}$ \\
\hline & Environmental regulation & $-0.272^{\star \star \star}$ & $-0.126^{\star \star \star}$ & $-0.126^{\star \star \star}$ \\
\hline & Population scale & $1.064^{\star \star \star}$ & $0.750^{\star \star \star}$ & $0.731^{\star \star \star}$ \\
\hline \multirow{5}{*}{ Estimation results of linear regression model } & Economic growth & $0.657^{\star \star \star}$ & $0.499^{\star \star \star}$ & $0.676^{\star \star \star}$ \\
\hline & Energy structure & $0.167^{\star \star \star}$ & $0.358^{\star \star \star}$ & $0.479^{\star \star \star}$ \\
\hline & Energy intensity & $0.610^{\star \star \star}$ & $0.257^{\star \star \star}$ & $0.250^{\star \star \star}$ \\
\hline & Environmental regulation & $-0.246^{\star \star \star}$ & $-0.118^{\star \star \star}$ & $-0.102^{\star \star \star}$ \\
\hline & Population scale & $1.050^{\star \star \star}$ & $2.249^{\star \star \star}$ & $0.632^{\star \star \star}$ \\
\hline
\end{tabular}
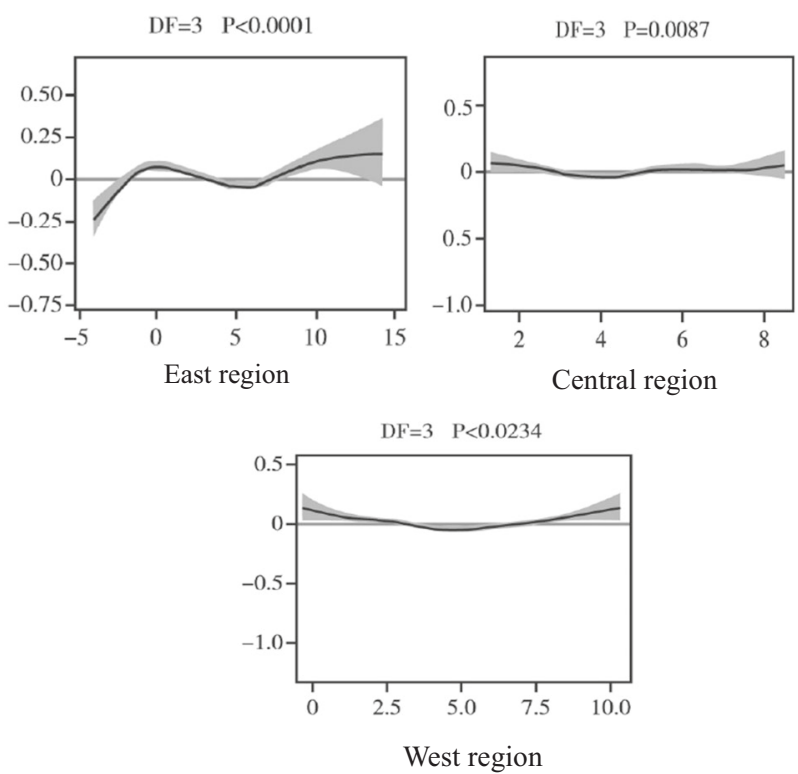

Fig. 2 The nonlinear impact of CED on CDE in three regions. The nonlinear impact of CED on CDE shows an "M-shape" curve in the eastern region. The nonlinear impact of CED on CDE shows a gentle "W-shape" curve in the central region. The nonlinear impact of CED on CDE shows a " $U$ shaped" curve in the western region.

figure shows that $\mathrm{CED}$ in the earlier stage is conducive to economic growth, while the promoting effect of CED on economic growth gradually weakened in the later period. The primary cause is abundant hydropower resources in the western region with the characteristics of low price, clean environmental protection, and renewable. The CED represented by hydropower can reduce the cost of economic growth. In the long term, the realization of sustainable economic growth in the western region mainly depends on technological progress. The energy consumption is gradually decreasing as a share of producers' costs, and the contribution of CED to economic growth is slowly decreasing.

The impact of CED on regional CDE. This section uses Eq. (10) to regression estimate and the analysis of the results. Among them, Table 3 shows the consequences of the linear impact of "a non-parametric additive regression model", and Fig. 2 shows the consequences of nonlinear implications.
The results of linear impact. The impact coefficients of CED on $\mathrm{CDE}$ in "the eastern, central, and western regions" are all positive, but the numerical values are relatively small. This means that CED has not significantly reduced CDE. The primary cause is that the $\mathrm{CE}$ production scale is relatively small, and energy consumption still mainly relies on fossil fuels (e.g., petroleum and coal). Under the background of the rapidly growing demand for energy consumption, fossil fuel consumption has grown much faster than the consumption of $\mathrm{CE}$, resulting in the offset of the $\mathrm{CDE}$ reduction. The effect coefficients of environmental regulation on CDE in "the eastern, central and western regions" are negative, which means that local governments at all levels should strengthen environmental governance efforts to decrease CDE.

The results of nonlinear impact. Figure 2 shows the nonlinear effect of CED for the eastern region on CDE appearing as an "Mshape" curve and passed the significance level test of $1 \%$. These indicate that CED in the early stage did not exert an essential role in decreasing CDE. The east region is economically well developed and needs to expend a lot of fossil fuels in China. Fossil fuels consumption has brought a lot of CDE, and governments at all levels in China have realized the importance and urgency of reducing fossil fuels and expanding the employ of CE. But because of the small-scale CE industry in the early term did not effectively reduce $\mathrm{CDE}$. In the medium stage, affected by the petroleum price in the world market, CED has fluctuated to a certain extent, affecting CDE. The production scale of $\mathrm{CE}$, in the long run, continues to expand, and its effect on CDE reduction is noticeable.

The nonlinear impact of CED for the central region on $\mathrm{CDE}$ reduction shows a gentle "W-shape" curve. This means that CED is conducive to CDE reduction in the earlier stage, while CED in the later stage has not exerted a significant role in decreasing CDE. This is mainly because, to alleviate energy shortage and control CDE aroused by fossil energy consumption, the central region focuses on CED represented by hydropower. In the early stages, hydropower can effectively alleviate energy shortages and decrease CDE from the consumption of fossil energy. However, with further economic growth, the growth of energy consumption exceeds CED. The hydropower resources in the central region are limited, making it difficult to highlight the role of CED in decreasing CDE.

The nonlinear impact of CED for the west region on CDE displays a "U-shaped" curve, namely the function of CED in decreasing CDE is mainly in the early stage. There are a lot of hydropower resources in the western region, which can fill the bill 
Table 4 "Residual sum of squares" of "the non-parametric additive regression model" and "the linear regression model".

\begin{tabular}{|llllll} 
& \multicolumn{2}{c}{ Dependent variables: CDE } & & & Dependent variables: economic growth \\
\cline { 2 - 3 } \cline { 5 - 6 } & $\begin{array}{l}\text { "Residual sum of squares" } \\
\text { of "the linear regression } \\
\text { model" }\end{array}$ & $\begin{array}{l}\text { "Residual sum of squares" of } \\
\text { "the non-parametric additive } \\
\text { regression model" }\end{array}$ & & $\begin{array}{l}\text { "Residual sum of squares" } \\
\text { of "the linear regression } \\
\text { model" }\end{array}$ & $\begin{array}{l}\text { "Residual sum of squares" of } \\
\text { "the non-parametric additive } \\
\text { regression model" }\end{array}$ \\
\hline East region & 33.940 & 8.627 & 79.623 & 8.607 \\
Central region & 30.303 & 6.170 & 29.989 & 7.509 \\
West region & 86.234 & 8.635 & 24.520 & 9.639 \\
\hline
\end{tabular}

Table 5 The linear effect of CED on regional economic growth (dependent variables: GDP).

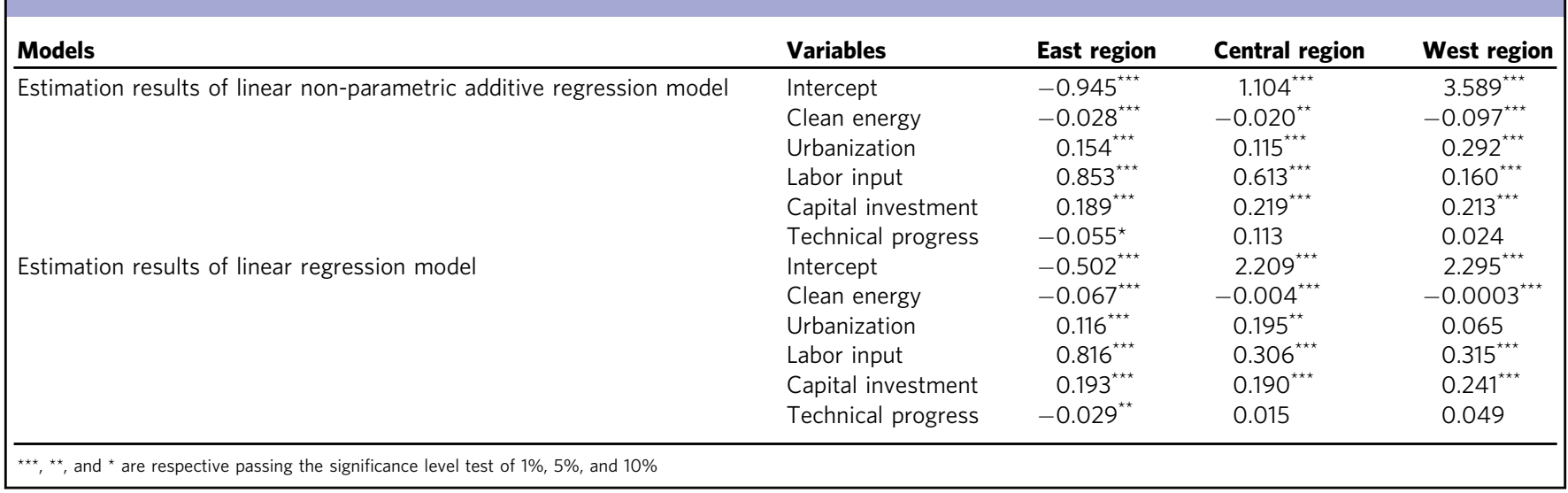

of industrial development and people's life, and help decrease the consumption of fossil fuels and CDE. The economic growth not only promotes the continuous expansion of the industrial scale but also improves the EI of residents' lives in the long run, which has contributed to swift growth in the quantity of energy consumed. The swift growth in the total quantity of energy consumed exceeds the expansion of CE, which makes the impact of CED on CDE reduction gradually eroded.

The fitting effect test. To test the validity of "a non-parametric additive regression model" estimation results, "the linear panel regression model" is employed for regression estimation. "The residual sum of squares" of the two models are shown in Table 4. The estimated results of "a non-parametric additive regression model" are significantly smaller than "a traditional linear panel regression model." These indicate that "a non-parametric additive regression model" fits better.

The robustness test. Since the non-parametric estimation results are sensitive to data changes, data slightly adjusted may bring about the non-parametric estimation results to change significantly. Therefore, the robustness of the estimation results in this research is tested through the parameter estimation results.

According to Peter et al. (2020), this research employs GDP instead of average per person GDP to assess the economic growth. The regression results with GDP as the dependent variables are compared with the previous regression results with per capita GDP as the dependent variables to inspect the robustness of the above estimation results. The regression coefficient symbols of independent variables in Table 5 are consistent with those of independent variables in Table 2, which shows that the estimation results of the models are robust.

To inspect the robustness of CED on the estimation results of the CDE model. According to Chen et al. (2013), this paper uses nominal GDP per capita for regression estimation (Table 6).
The explanatory variables symbols that impact CDE in Table 6 are in line with the consequences in Table 3. These show that the consequences of CED on CDE in Table 3 are robust.

\section{Conclusions and policy recommendations}

According to the panel data of 30 provinces in China composed of accurate data from 1979 to 2016 and forecast data from 2017 to 2030, this research employs "a non-parametric and additive regression model" in exploring the linear effect and nonlinear effect of CED on regional economic growth and CDE. The linear impact results show that CED does not fulfill the function of CDE reduction in the three regions. Moreover, governments at various levels have invested a lot of monetary funds and implemented tax relief on CED, which has increased the economic burden and hindered the economic growth of the three regions. In fact, under the market economy condition, the environment of economic growth is complex and changeable. CED should not only follow the market rules but also be affected by the government's fiscal and tax policies. Therefore, the role of CED in various phases of China's economic development is different. Therefore, the research on the part of $\mathrm{CED}$ on regional $\mathrm{CDE}$ reduction and economic growth is closer to the reality of China's economic growth and has more practical significance during the various phases. The main nonlinear conclusions and policy recommendations obtained are as follows: First, CED has an "M-shaped" nonlinear impact on CDE in the east of China. That is, CED has not fulfilled the function of CDE reduction in the early phase; the macroeconomic impact caused fluctuation in CED in the medium stage, and its effect on CDE also fluctuates slightly; CED contributes to $\mathrm{CDE}$ reduction in the long run. Therefore, to improve the $\mathrm{CDE}$ reduction impact of $\mathrm{CE}$ in the early phase, the central and local governments should have some preferential policies in promoting the rapidly growing CE industry.

Second, CED has a gentle "U-shaped" nonlinear effect on CDE in central China. The CDE reduction effect of $\mathrm{CE}$ resources is 
Table 6 The linear impact of CED on regional CDE.

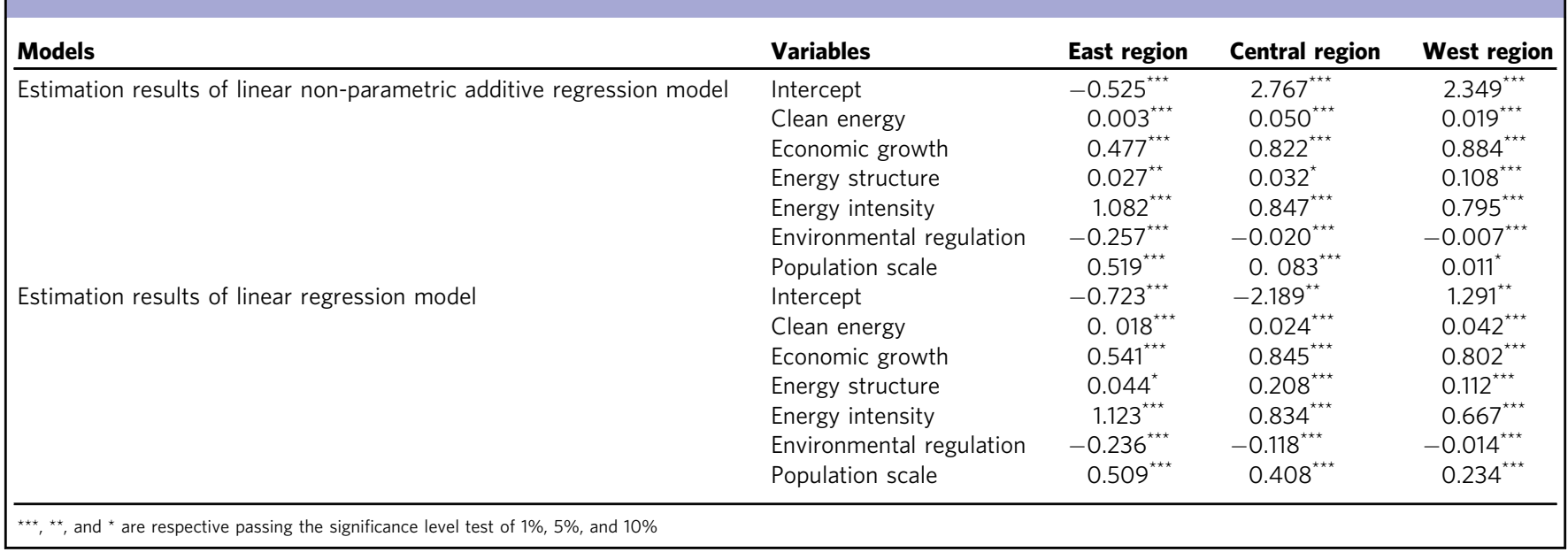

challenging to be realized in the later phase. The main reason is the scattered resources, high cost, and small production scale of $\mathrm{CE}$ in central China. The central region should expand its $\mathrm{CE}$ supply and reduce fossil fuel use in the long term.

Third, CED has a "U-shaped" nonlinear impact on CDE in the west of China. The abundant water energy resources in western China are the main reason and have been actively developing hydropower for many years. However, hydropower resources cannot be unrestricted to development. In the long run, the growth in hydropower production will gradually slow, while the rapid increase in fossil fuel consumption caused by economic growth will negate the CDE reduction effect of CE. The western region should increase $\mathrm{CE}$ consumption to reduce fossil fuel consumption and CDE.

Fourth, CED has a gentle "W-shaped" nonlinear impact on economic growth in the east of China. CED did not promote economic growth in the early phase. Local governments invested a lot of monetary funds and adopted some preferential policies (e.g., abatement of tax and free of duty), which promoted CED. However, substantial financial investment and abatement of tax and free of responsibility have increased the financial burden. The local governments should lead the energy market to enlarge the consumption of CE and avoid being overly reliant on government investment to stimulate CED.

Fifth, the nonlinear effect of CED on economic growth is a gentle "W-shaped" in the central region. CED did not fulfill the function of promoting economic growth in the early phase; the role of CED in promoting economic growth gradually emerged in the later stage. The main reason is that the central region is suitable for CE development. However, the distribution of resources is scattered, and the scale of production enterprises is small. Local governments ought to strongly encourage the growth of $\mathrm{CE}$ producers, increase capital investment, and reduce taxes.

Sixth, the nonlinear effect of CED on economic growth is "inverted U-shaped" in the western region. Substantial investment in CED boosted economic growth in the early stage. The sustainable economic growth of the region of the west in the long run mainly depends on technological progress and innovation. The energy consumption costs as a share of producers' production costs will gradually decline. Thus the contribution of the growth of the $\mathrm{CE}$ industry to economic growth will decline progressively. Therefore, the western region should pay attention to talent training and technological innovation to promote sustained economic growth.

\section{Data availability}

All data analyzed are contained in the paper.

Received: 7 April 2021; Accepted: 22 December 2021; Published online: 19 January 2022

\section{References}

Bravo OC, Marin A (2011) R\&D and productivity: a two way avenue? World Dev 39(7):1090-1107

Buja TH, Tibshirani R (1989) Linear smoothers and additive-models. Ann Stat 17(2):453-510

Cao J (2009) The low-carbon economic development mode: China's carbon tax policy design and CGE model analysis. J Financ Res. 354(12):19-29. (in Chinese)

Chen C, Li YP, Huang GH (2013) An inexact robust optimization method for supporting carbon dioxide emissions management in regional electric-power systems. Energy Econ 40(11):441-456

Chen S (2009) Energy consumption, carbon dioxide emission and sustainable development in Chinese industry. Econ Res. 4:48-59. (in Chinese)

Costanza R et al. (2014) Development: time to leave GDP behind. Nature 505:283-285

Dogan E, Seker F (2016) Determinants of $\mathrm{CO}_{2}$ emissions in the European Union: the role of renewable and non-renewable energy. Renew Energ 94:429-439

Ehrlich PR, Holden JP (1971) Impact of population growth. Science 171:1212-1217 Ehrlich PR, Holden JP (1972) One dimensional economy. Bull Atom Sci 16:18-27 Engle R, Hendry D, Richard J (1983) The exogeneity. Econometrica 51(2):277-304 Hausman JA (1978) Specification tests in econometrics. Econometrica 46(6):1251-1271 Inglesi LR (2016) The impact of renewable energy consumption to economic growth: a panel data application. Energy Econ 53:58-63

Kahia M, Kadria M, Assa SB (2016) What impacts of renewable energy consumption on $\mathrm{CO}_{2}$ emissions and the economic and financial development? A panel data vector autoregressive (PVAR) approach. In 7th international renewable energy congress (IREC), institute of electrical and electronics engineers (IEEE) 3:22-24

Kathia M, Aissa MB, Lanouar C (2017) Renewable and non-renewable energy useeconomic growth nexus: the case of mena net oil importing countries. Renew Sustain Energy Rev 71:127-140

Kocak E, Sarkgünesi A (2017) The renewable energy and economic growth nexus in Black sea and Balkan countries. Energy Policy 100:51-57

Li J, Liu H, Du K (2019) Does market-oriented reform increase energy rebound effect? Evidence from China's regional development. China Econ Rev 56:101-113

Lin BQ, Li JL (2014) Quantitative evaluation of Chinese renewable energy policy based on recursive stochastic dynamic programme. J Econ Res. J 4:89-103. (in Chinese)

Lin BQ, Zhu JP (2017) Energy and carbon intensity in China during the urbanization and industrialization process: a panel VAR approach. J Clean Prod $168: 780-790$ 
Lu WB, Qiu TT, Du L (2013) A study on influence factors of carbon emissions under different economic growth stages in China. J Econ Res. J 4:106-118. in Chinese

Lu X, Xu CX (2019) The difference and convergence of total factor productivity of inter-provincial water resources in China based on three-stage DEA-Malmquist index model. Sustain Comput-Infor 22:75-83

Miao Z, Baležentis T, Tian Z, Shao S, Geng Y, Wu R (2019) Environmental performance and regulation effect of China's atmospheric pollutant emissions: evidence from Three regions and ten urban agglomerations. Environ Resour Econ 74(1):211-242

Nan Z et al. (2019) A roadmap for China to peak carbon dioxide emissions and achieve a $20 \%$ share of non-fossil fuels in primary energy by 2030. Appl Energ 239:793-819

National Bureau of Statistics of China (1979-2016) China statistical yearbook. China Statistics Press, Beijing, China (in Chinese)

National Bureau of Statistics of China (2019) China statistical yearbook. China Statistics Press, Beijing, China (in Chinese)

Nie YY, Li QH, Wang EC, Zhang TY (2019) Study of the nonlinear relations between economic growth and carbon dioxide emissions in the Eastern, Central and Western regions of China. J Clean Prod 219:713-722

Peter W, Yong T, Jorge A, Abdollah HV (2020) Business environment drivers and technical efficiency in the Chinese energy industry: a robust bayesian stochastic frontier analysis. Comput Ind Eng 144(6):164-181

Pokki H, Virtanen J, Karvinen S (2018) Comparison of economic analysis with financial analysis of fisheries: application of the perpetual inventory method to the Finnish fishing fleet. Mar Pol 95:239-247

Qiu TW, Luo BL et al. (2020) Do land renting-in and its marketization increase labor input in agriculture? Evidence from rural China. Land Use Pol 99(12):48-62

Shao S, Li X, Cao JH(2019a) Urbanization promotion and haze pollution governance in China. J Econ Res 2:148-165. in Chinese

Shao S, Chen Y, Li K, Yang LL (2019b) Market segmentation and urban $\mathrm{CO}_{2}$ emissions in China: evidence from the Yangtze River Delta region. J Environ Manag 248:193-204

Stone CJ (1985) Additive regression and other nonparametric models. Ann Stat 64:689-705

Tian X, Bai FL, Jia JH, Liu Y, Shi F (2019) Realizing low-carbon development in a developing and industrializing region: Impacts of industrial structure change on $\mathrm{CO}_{2}$ emissions in southwest China. J Environ Manage 233:728-738

Wang Q, Wang SS (2019) A comparison of decomposition the decoupling carbon emissions from economic growth in transport sector of selected provinces in eastern, central and western China. J Clean Prod 229(20):570-581

Wen L, Shao HY (2019) Influencing factors of the carbon dioxide emissions in China's commercial department: a non-parametric additive regression model. Sci Total Environ 668:1-12

World Bank (2019) $\mathrm{CO}_{2}$ emissions. https://data.worldbank.org/indicator/ EN.ATM.CO2E.KT? view $1 / 4$ chart

Wu S, Han HY (2020) Sectoral changing patterns of China's green GDP considering climate change: an investigation based on the economic input-output life cycle assessment model. J Clean Prod 251:119-124

Wu DM (1974) Alternative tests of independence between stochastic regressors and disturbances: finite sample results. J Econ Soc 42(3):529-546

Yang C, Poon JPH (2009) A regional analysis of China's green GDP. Eurasian Geogr Econ 50:547-563

Yang XJ, Hu HJ, Tan TW, Li JY (2016) China's renewable energy goals by 2050. Environ Dev 20:83-90

Yang L, Yang YT (2019) Evaluation of eco-efficiency in China from 1978 to 2016: Based on a modified ecological footprint model. Sci Total Environ 662:581-590
York R, Rosa EA, Dieta T (2003) STIRPAT, IPAT and Impact: analytic tools for unpacking the driving forces of environmental impacts. Ecol Econ 46:351-365

Zeng S, Jiang C, Ma C, Su B (2018) Investment efficiency of the new energy industry in China. Energy Econ 70:536-544

Zhang XD, Liu XY (2015) Study on carbon taxation and developing analyses based renewable energy on OLG-CGE model growth and welfare effects China industrial economics. China Ind Econ 324(3):19-30. (in Chinese)

Zoundi $\mathrm{Z}$ (2017) $\mathrm{CO}_{2}$ emissions, renewable energy and the environmental Kuznets curve, a panel cointegration approach. Renew Sustain Energy Rev 72:1067-1075

\section{Acknowledgements}

Research works in this paper are financially supported by the National Social Science Fund of China (Grant No. 17BZZ046).

\section{Author contributions}

All authors have contributed to this research equally and agree to be held accountable for the accuracy and integrity of this research.

\section{Competing interests}

The authors declare no competing interests.

\section{Ethical approval}

This article does not contain any studies with human participants performed by any of the authors.

\section{Informed consent}

This article does not contain any studies with human participants performed by any of the authors.

\section{Additional information}

Correspondence and requests for materials should be addressed to Weiping Jia.

Reprints and permission information is available at http://www.nature.com/reprints

Publisher's note Springer Nature remains neutral with regard to jurisdictional claims in published maps and institutional affiliations.

(c) (i) Open Access This article is licensed under a Creative Commons BY Attribution 4.0 International License, which permits use, sharing adaptation, distribution and reproduction in any medium or format, as long as you give appropriate credit to the original author(s) and the source, provide a link to the Creative Commons license, and indicate if changes were made. The images or other third party material in this article are included in the article's Creative Commons license, unless indicated otherwise in a credit line to the material. If material is not included in the article's Creative Commons license and your intended use is not permitted by statutory regulation or exceeds the permitted use, you will need to obtain permission directly from the copyright holder. To view a copy of this license, visit http://creativecommons.org/ licenses/by/4.0/.

(C) The Author(s) 2022 